\title{
A Study on Residents' Catering Consumption Behavior in Shiyan City under the Background of Mobile E-commerce
}

\author{
Lei Guo ${ }^{a}$, Tianling Li ${ }^{b}$, Ruijiang Nan ${ }^{c}$, Na Zheng ${ }^{d}$ \\ School of Economics and Management, Hubei Automotive Industries Institute, Shiyan, China \\ agl0327@163.com, b451205866@qq.com, c48522224@qq.com, d438417767@qq.com
}

Keywords: Mobile e-commerce, catering industry, consumer behavior, Shiyan City Introduction

\begin{abstract}
With the rapid development of network technology, the continuous expansion of the scale of catering industry and the upgrading of consumer demand, the penetration and integration of mobile e-commerce in the catering industry has been continuously strengthened. This brings new opportunities for the development of the catering industry in Shiyan, and also has various impacts on the behavior of consumers. Taking the residents of Shiyan City as the survey object, it analyzes and summarizes the characteristics of catering consumption behavior under the background of mobile e-commerce in order to provide reference for the targeted product development and marketing under the background of mobile e-commerce in Shiyan City.
\end{abstract}

\section{Introduction}

In recent years, with the development of information technology and the extensive use of the Internet, mobile e-commerce has achieved rapid development and gradually penetrated into people's daily lives, affecting consumer behavior in many ways. Even under the powerful impact of the Internet, the economic performance of catering economy, also known as "the tongue economy", remains fairly stable. The relevant data show that the Chinese catering industry has been transforming since 2012, and the national revenue of the catering industry has remained at 3.9 trillion yuan in 2017, and the double-digit growth has resumed for three consecutive years, highlighting the strong mass consumption power of the catering market.

When the traditional catering service approach collided with the new needs of consumers, mobile e-commerce catering came into being. The operation of the catering industry is becoming increasingly diverse, including the take-away catering brands, $\mathrm{O} 2 \mathrm{O}$ platforms, catering management service providers and new food media. In many first-tier cities, the catering market is relatively saturated and the income growth is slowing down. However, the proportion of catering consumption in small and medium-sized cities has increased, which means the mobile Internet will bring more opportunities for the development of catering industry in small and medium-sized cities.

As a four-tier city, Shiyan City, along with the changes in the consumption environment and the unchanging passion for delicacy, eating out has become more popular for people in Shiyan. In light of this, studying the characteristics of catering consumption behavior of residents in Shiyan City under the background of mobile e-commerce will help catering enterprises to better understand the market demand under the new situation, and transform their catering business accordingly, thereby promoting the sustained and stable development of catering industry in Shiyan City.

\section{Survey design and implementation}

A sample survey of the consumption behavior of residents in Shiyan City under the mobile ecommerce background was conducted both offline and online. A total of 200 questionnaires were distributed and 186 valid questionnaires were collected, with an effective rate of $93 \%$. The content of the questionnaire mainly includes the demographic characteristics of the respondent, the frequency of eating out, the source of information, taste preference, the preference of payment modes, and the willingness of ordering food via mobile terminal. The offline survey mainly distributes 
questionnaires randomly among catering enterprises, schools, and streets in the downtown area of Shiyan, while the online survey is mainly conducted through questionnaire surveying platforms or forwarding links. Finally, the survey data is input into Excel, and then is processed and analyzed by SPSS18.0 to draw relevant conclusions.

\section{Analysis of survey results}

\subsection{Consumer demographics}

In terms of gender, women account for $45.3 \%$, and the percentage of men is slightly higher, accounting for $54.7 \%$. This is in line with the current situation where men are more inclined to eat out and are not willing to cook at home. In terms of age, the sample is mainly concentrated between 18 and 25-year- olds, accounting for $69 \%$. It can be seen that the current consumption of food and beverage on the mobile e-commerce platform is dominated by young groups. In education degree, people with undergraduate degree and above account for $75.4 \%$, while the proportion of people with high school diploma and below is relatively small, only $8.4 \%$, which shows that the higher the education level, the stronger the ability to accept new things, and the more likely for them to use mobile e-commerce platform for consumption. In terms of monthly income, the respondents whose income level is below 2,000 yuan and beween 3001-5000 yuan account for more than 63\%, indicating that the restaurant goers in Shiyan City are mainly students and working-class people, who like to pursue fast, economical and fashionable dining.

\subsection{Analysis of characteristics of catering consumption behavior}

\subsubsection{Frequency analysis of dining out}

A survey of dining out frequency of citizens shows that about $37.3 \%$ of people eat out three times or more per week, $46.7 \%$ of them eat out $1-2$ times a week, and $16 \%$ may go out for dinner $1-2$ times a month. It can be seen that dining out is quite popular among people in Shiyan, and it is almost a necessary part of their life. This also indicates that there is still unlimited market space for the development of the catering industry in small and medium-sized cities.

\subsubsection{Catering information collection and analysis}

Due to the popularity of mobile phones, IPAD and other mobile terminals, $77.3 \%$ of residents will use the mobile APP or group purchase network to search for suitable restaurants and food before going out to eat. Among them, group purchase network represented by Meituan, ele.me, and Public Comment are most commonly used, and the sum of the three uses is close to $90 \%$. This shows that the mobile e-commerce platform has played an increasingly important role in the collection of people's food consumption information, and has become a common channel for collecting information.

\subsubsection{Food taste preference analysis}

A variety of cuisines are presented in the mobile e-commerce platforms. According to a survey of the taste preference of Shiyan residents (Table 1), the common foods popular among people in the mobile e-commerce platforms are mainly hot pot (77.4\%), buffet (65.3\%), local specialties (58.7\%), barbecue (45.3\%), etc., fully demonstrating the enthusiasm of the Shiyan residents for diversified diets, and it also reveals the diversified development status of the catering industry in Shiyan. This is partly due to the location of the city. Shiyan is located at the junction of Hubei, Henan, Shaanxi and Anhui province. The diversification of culture has created a diversified diet. The reason why hot pot is most favored by the public is because of the unique geographical environment and special climatic conditions of Shiyan City (such as the temperature difference between morning and evening, the humidity, etc) and the multiculturalism commonly seen in immigrant cities. Moreover, hot pot suits Chinese people's eating habits: eating in groups in a carefree way. Besides, hot pot consumption is suitable for all stages ${ }^{[3]}$. The buffet is also very popular, mainly because it has the advantages of 
convenient access, diverse tastes, and strong autonomy. At the same time, it serves as a casual and simple meal to cater to the needs of consumers in pursuit of fashion.

Table 1 Taste preference

\begin{tabular}{lll}
\hline Types of diet & Frequency & Ranks \\
\hline Buffet & 65.3 & 2 \\
Hotpot & 77.4 & 1 \\
Western food & 29.2 & 5 \\
Local specialties & 58.7 & 3 \\
Japanese and Korean cuisine & 10.6 & 7 \\
Barbeque & 45.3 & 4 \\
Desserts and drinks & 22.7 & 6 \\
Others & 12 & 7 \\
\hline
\end{tabular}

\subsubsection{Analysis of factors affecting dining out}

The survey showed that food taste (85.3\%), food safety $(71.3 \%)$, product price $(68 \%)$, dining atmosphere (66.7\%), and service quality (57.4\%) were the main factors affecting the Shiyan citizens when they eat out. As is stated previously, the respondents are relatively educated, so they pays more attention to taste, safety, service, environment, etc. when dining out. At the same time, because the monthly income is relatively low, they are also sensitive to the price of the meal.

\subsubsection{Analysis of payment modes}

Among the current payment modes, consumers in Shiyan City are more inclined to use third-party platforms for mobile payment (78.7\%), much higher than even the sum of credit card payment (18.7\%) and cash payment (2.6\%). Further investigation found that $63.9 \%$ of the actual payments were made by mobile payment, and $36.1 \%$ paid by cash or credit card. The results indicate that people not only prefer but also are accustomed to the payment via mobile terminals, and we are entering the "cashless" era. Fundamentally, third-party payment will become a trend in the future as it simplified transaction operations, lower transaction costs, and meets consumer preferences in online ordering.[4] More than 83\% of consumers in the survey have basically adopted a positive attitude towards the application of mobile e-commerce in catering. They believe that it is very promising. Only a few people are not optimistic about the application of mobile e-commerce.

\subsubsection{Willingness and perception of dieting via mobile terminals $\square$}

From the statistical data(Table 2), consumers have a strong preference to the mobile e-commerce platform (the average is above 3.5). The details are as follows: First, the frequency of purchases by residents of Shiyan City through the mobile terminal is high (mean value 4.3), indicating that residents have begun and gradually become accustomed to the form of online consumption. The reason is that on the one hand, more and more catering enterprises put catering information into the mobile e-commerce platform, so consumers can use these platforms to complete the purchase behavior flexibly and autonomously; on the other hand, third-party mobile e-commerce platforms often initiate some promotional activities, such as group purchases, discounts and coupons, to attract consumers, which stimulates their desire to buy. Second, the citizens in Shiyan pay great attention to online sales and evaluation (mean 4.12) when making food and beverage decisions. It can be seen that the mobile e-commerce platform has better solved the problem of information asymmetry between consumers and merchants in the past, so that catering consumers can obtain business information more comprehensively and quickly, which is conducive to consumer decision-making; third, due to the e-commerce platform Involved, more and more catering consumers are accustomed to online ordering (mean 3.93). This highlights a trend of fast, fashionable and distinctive national catering consumption. Fourth, citizens in Shiyan prefer to complete order purchases (mean 3.89) on well-known platforms or apps. It shows that catering consumers value the information channels, product quality, after-sales service, etc. Finally, consumers agree that the price comparison can be made through the mobile e-commerce platform, so that they can get their desired products at a 
satisfactory price (mean 3.72).

Table 2 Willingness and perception of dieting via mobile terminals

\begin{tabular}{ll}
\hline Survey items & Mean \\
\hline I prefer to order on a well-known website or app. & 3.89 \\
I will make purchasing decisions based on online sales and reviews. & 4.12 \\
I will use the mobile terminal for price comparison. & 3.62 \\
I prefer the mobile terminal for it is practical and convenient. & 4.3 \\
The mobile terminal makes me more willing to order via it & 3.93 \\
\hline
\end{tabular}

\section{Conclusion}

Based on the survey and analysis, the following conclusions can be drawn:

\subsection{The demand for catering industry is strong, and the youth group has become the main consumption power of Shiyan City}

Eating is the top priority in the lives of the Chinese people. About $80 \%$ of the Shiyan residents in the survey will go out to eat once or twice a week, reflecting the strong demand for food and beverage consumption, and the catering industry in Shiyan City has great potential. The consumer groups who go out to eat are mainly young people aged 18-25, indicating that "post-90s" and "post00s" have become the main consuming power of the catering industry in Shiyan City. This conclusion is in accordance with the "2017 Food Consumption Report" issued by the China Cuisine Association. The report pointed out that among the people who consume food and beverage, the younger generation has become the new main force, among which "post-90s" and "post-00s" contributed orders of about $50 \%$ of food and beverage consumption. This is because, on the one hand, young people tend to try new things, and they are "native" people in the internet age, armed with mobile phones, laptops, pads and other mobile terminals. On the other hand, they are more innovative, with more social activities, and they pursue a personalized, diverse and convenient lifestyle.

\subsection{Catering consumption is highly dependent on mobile terminals, and the reputation of e- commerce platform has a greater impact on consumption decisions}

The citizens in Shiyan have great dependence on mobile e-commerce in the process of food consumption. Before making decision for food consumption, they usually collect food information through third-party platforms, and most of them will make purchasing decisions based on online sales volume and evaluations. They will use the mobile terminal to compare prices to obtain more favorable prices. Once the decision is made, they tend to place their orders and pay for the bills through the mobile terminal. It can be seen that mobile e-commerce has penetrated into the hearts of food consumers, and good word-of-mouth is crucial for the development of the catering industry in Shiyan City in the context of mobile e-commerce, because consumers are more inclined to believe in the evaluation and comments from other consumers on third-party Internet platforms and social platforms. For example, in terms of access to restaurant information, consumer evaluation on thirdparty platforms is exerting increasing influence on consumers' decision making.

\subsection{Food consumers pursue quality experience and are sensitive to the price/performance ratio of products}

When consumers go out to eat, the main factors of concern are food taste, safety, price, atmosphere, service quality, etc., reflecting the pursuit of high-quality experience and clean and hygienic environment. The food safety factor ranks second, indicating that the food safety, besides food taste, has always been the focus of the people. They are paying more and more attention to food safety, and their tolerance for safety problems is virtually zero. How to assure food safety is not only a test of the government and enterprises, but also a social problem that all of us are involved. On the other hand, due to the limited income, consumers, when eating out, are sensitive to the 
price/performance ratio of products, so product prices become an important factor. When it comes to payment, it is interesting to note that with the progress of the times and the rapid development of information, the acceptable payment modes and ordering channels provided by catering enterprises have also become the concern of consumers.

\subsection{Cash payment is losing its appeal while mobile payment is getting popular}

According to the survey, the current cash payment and credit card payment are losing their appeal, and more and more consumers make their payment via third-party platforms such as WeChat and Alipay. With the rapid increase in the number of mobile payment users, mobile payment has penetrated into various scenarios of people's lives. Compared with credit card, third-party payment platforms are favored by catering industry as well as customers because of their convenience and low operation fees.).

\section{Acknowledgments}

I would like to thank the 2017 Wudang Culture Research and Communication Center Open Fund Project (17wdjd016) and the 2017 HUAT College Student Innovation Training Program Project (SJ201778) for their support.

\section{References}

[1] XueZhang. "Small and beautiful" has become a new trend in the development of food and beverage industry. http://www.ce.cn/Xwzx/gnsz/gdxw/201707/12/t20170712_24165979.shtml, (2017-07-12).

[2] Li-ling Wen. The study on catering consumption behavior of residents of Nanning. Guangxi University, 2012. (In Chinese)

[3] Li-ling Wen. Surey on residengt catering buying behaviors in Nanning. Enterprise Science and Technolgy\& Development, vol.17(2012), pp.:7-8. (In Chinese)

[4] Chang-cheng $\mathrm{Li}$, Ju Wang. "Investigation on the food and beverage consumption behavior of residents in the "Internet +" era in Yongchuan Distric". knowledge economy, vol. 22, 2016, pp. 74+76. (In Chinese)

[5] Chinese Cuisine Association. A survey of food consumption in 2017. http://www.ccas.com.cn/Article/HTML/108593_3.html. (2018-1-18).

[6] Qian-nan Bu. Study on tourist consumption behavior under the background of mobile ecommerce. East China Normal University, 2016. (In Chinese)

[7] $\mathrm{Zi}$ - cheng $\mathrm{Li}$. Internet depth affects food and beverage consumption. International Business.2017-1 -9(A08). 